\title{
PLA2R1 promotes DNA damage and inhibits spontaneous tumor formation during aging
}

\author{
Anda Huna', Audrey Griveau', David Vindrieux', Sara Jaber', Jean-Michel Flaman', Delphine Goehrig', Lamia Azzi ,

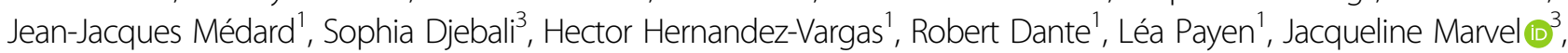 \\ Philippe Bertolino', Sébastien Aubert ${ }^{4}$, Pierre Dubus ${ }^{2,5}$ and David Bernard (iD)
}

\begin{abstract}
Although aging is a major risk factor for most types of cancers, it is barely studied in this context. The transmembrane protein PLA2R1 (phospholipase A2 receptor) promotes cellular senescence, which can inhibit oncogene-induced tumor initiation. Functions and mechanisms of action of PLA2R1 during aging are largely unknown. In this study, we observed that old Pla2r1 knockout mice were more prone to spontaneously develop a wide spectrum of tumors compared to control littermates. Consistently, these knockout mice displayed increased Parp1, a master regulator of DNA damage repair, and decreased DNA damage, correlating with large human dataset analysis. Forced PLA2R1 expression in normal human cells decreased PARP1 expression, induced DNA damage and subsequent senescence, while the constitutive expression of PARP1 rescued cells from these PLA2R1-induced effects. Mechanistically, PARP1 expression is repressed by a ROS (reactive oxygen species)-Rb-dependent mechanism upon PLA2R1 expression. In conclusion, our results suggest that PLA2R1 suppresses aging-induced tumors by repressing PARP1, via a ROS-Rb signaling axis, and inducing DNA damage and its tumor suppressive responses.
\end{abstract}

\section{Introduction}

Aging is a major risk factor for most human cancers ${ }^{1}$. It can be modeled in mice that spontaneously develop tumors during aging without prior exposure to other risk factors such as radiation, cigarette smoke, or alcohol ${ }^{2}$. Nevertheless, the ways in which aging impacts cancer formation has seldom been studied.

Reactive oxygen species (ROS) and subsequent DNA damage accumulates during aging ${ }^{3}$. They have been shown to exert both pro- or anti-tumoral effects, but still their role during aging and the increased risk of spontaneous tumor formation are largely unknown ${ }^{4-9}$. DNA

\footnotetext{
Correspondence: David Bernard (david.bernard@lyon.unicancer.fr)

${ }^{1}$ Centre de Recherche en Cancérologie de Lyon, Inserm U1052, CNRS UMR

5286, Centre Léon Bérard, Université de Lyon, Lyon, France

${ }^{2}$ INSERM U1053 Bordeaux Research in Translational Oncology, University of

Bordeaux, Bordeaux Cedex, France

Full list of author information is available at the end of the article

These authors contributed equally: Anda Huna, Audrey Griveau, David

Vindrieux

Edited by M. Agostini.
}

damage is expected to mainly exert pro-tumoral effects by promoting genomic instability and aneuploidy induction $^{7,10-13}$, still it can also exert anti-tumoral effects by fueling aneuploidy ${ }^{13}$ and by induction of DNA damage signaling resulting in p53 activation, senescence, and apoptosis $^{4-6}$.

PLA2R1 belongs to the mannose receptor family that can bind several matrix components, some sugar or several sPLA2 (secretory PLA2). Though little is known about the functional role of the transmembrane protein PLA2R1 in the cancer and aging contexts ${ }^{14,15}$, it was shown to increase ROS production and DNA damage accumulation leading to different cellular responses like cell death and cellular senescence ${ }^{16-18}$, and to different organismal outcome like limiting tumor formation during RAS-induced skin carcinogenesis ${ }^{19}$ and tumor growth ${ }^{20,21}$ as well as promoting some marks of premature aging in a murine model of progeria ${ }^{22}$.

In this study, we aimed at deciphering and investigating the role of the PLA2R1 protein during aging-induced

\section{(c) The Author(s) 2021}

(c) (i) Open Access This article is licensed under a Creative Commons Attribution 4.0 International License, which permits use, sharing, adaptation, distribution and reproduction cc) in any medium or format, as long as you give appropriate credit to the original author(s) and the source, provide a link to the Creative Commons license, and indicate if changes were made. The images or other third party material in this article are included in the article's Creative Commons license, unless indicated otherwise in a credit line to the material. If material is not included in the article's Creative Commons license and your intended use is not permitted by statutory regulation or exceeds the permitted use, you will need to obtain permission directly from the copyright holder. To view a copy of this license, visit http://creativecommons.org/licenses/by/4.0/. 
tumor formation using aged mice, normal human cells and large human datasets.

\section{Materials and methods}

\section{Cell culture and treatments}

MRC5 normal human fibroblasts (ATCC) and virusproducing GP293 cells (Clontech) were cultured in Dulbecco's modified Eagle's medium containing GlutaMAX and supplemented with $10 \%$ fetal bovine serum (FBS) (Sigma-Aldrich) and 1\% penicillin/streptomycin. Cells were maintained at $37^{\circ} \mathrm{C}$ under a $5 \% \mathrm{CO}_{2}$ atmosphere and used within 10 passages after purchasing. Cells were tested for mycoplasma and if needed treated with Plasmocin (Invivogen) until they became mycoplasma-free before performing experiments. $N$-acetyl cysteine (NAC) and Trolox were purchased from Sigma-Aldrich and used at $500 \mathrm{nM}$ and $5 \mu \mathrm{M}$, respectively. $\mathrm{H}_{2} \mathrm{O}_{2}$ was added at $250 \mu \mathrm{M}$ for $1 \mathrm{~h}$.

\section{siRNA transfection}

For the siRNA knockdown experiments, an ON-TARGETplus nontargeting control pool-siCtrl (sequences UGGUUUACAUGUCGACUAA, UGGUUUACAUGUU GUGUGA, UGGUUUACAUGUUUUCUGA, UGGUUU ACAUGUUUUCCUA) or ON-TARGETplus siRNA SMARTpools targeting the Rb1 (GAACAGGAGUGCA CGGAUA, GGUUCAACUACGCGUGUAA, CAUUAAU GGUUCACCUUCGA, CAACCCAGCAGUUCGAUAU) (Dharmacon) were used at $15 \mathrm{nM}$. Reverse transfection was performed in six-well plates (cell density $5 \times 10^{5}$ per well), using a $0.1 \%$ solution of Dharmafect 1 transfection reagent (Thermo Scientific).

\section{Vectors, transfection, and infection}

The retroviral vectors encoding human PLA2R1, pLPCX/PLA2R1 for puromycin selection or pLNCX/ PLA2R1 for neomycin selection, have previously been described; ${ }^{16,19,21}$ pBABE/PARP1 (puromycin selection) was described in ref. ${ }^{23}$; ROS reporter pLPCx-roGFP2ORP1 was described in ref. ${ }^{24}$.

Virus-producing GP293 cells were transfected with vectors using the GeneJuice reagent according to the manufacturer's recommendations (Merck Millipore). Cells were transfected with the VSVg $(1 \mu \mathrm{g})$ and the retroviral vector of interest $(5 \mu \mathrm{g})$. Two days after transfection, the viral supernatant was mixed with fresh medium $(1 / 2)$ and hexadimethrine bromide $(8 \mu \mathrm{g} / \mathrm{ml}$; SigmaAldrich), and was then used to infect target cells for $6 \mathrm{~h}$. One day after infection, selection was started using puromycin at $500 \mathrm{ng} / \mathrm{ml}$ or/and neomycin at $100 \mu \mathrm{g} / \mathrm{ml}$.

\section{SA- $\beta$-galactosidase assay, crystal violet staining and growth curves}

For SA- $\beta$-galactosidase assay, cells were washed twice with PBS and fixed for $5 \mathrm{~min}$ in $2 \%$ formaldehyde $/ 0.2 \%$ glutaraldehyde. Cells were then rinsed twice in PBS and incubated at $37^{\circ} \mathrm{C}$ overnight in a SA- $\beta$ - Gal solution as previously described ${ }^{25}$. For crystal violet staining, cells were washed with PBS, fixed for $15 \mathrm{~min}$ in $3.7 \%$ formaldehyde, and then stained with $0.05 \%$ crystal violet solution. For growth curves, cells were seeded at the same density and counted at each passage. The population doubling was calculated at each passage using the following formula: $\mathrm{PD}=\ln$ (number of collected cells/number of plated cells)/ln2.

\section{ROS detection}

Cells stably expressing roGFP2-ORP1 were washed and incubated in HBSS with calcium, magnesium, and no phenol red (14025050, Thermo Scientific) for ROS detection. Fluorescence (Ex 405 and $488 \mathrm{~nm} / \mathrm{Em}$ $500-554 \mathrm{~nm}$ ) was monitored in live cells using the Operetta system. The Columbus software was used to quantify fluorescence intensity, and the ratio between $405 \mathrm{~nm}$ (oxidized state) and $488 \mathrm{~nm}$ (reduced state) was calculated, as previously described ${ }^{26}$.

\section{Reverse transcription and real-time quantitative PCR}

Total RNA was isolated by phenol-chloroform extraction on cells. The Maxima First cDNA Synthesis Kit (Life Technologies) was used to synthesize cDNA according to the manufacturer's instructions from $1 \mu \mathrm{g}$ of RNA. The reverse transcription (RT) reaction mixture was used at a dilution of $1 / 20$ as a cDNA template for quantitative PCR (qPCR) analysis. TaqMan qPCR analyses were carried out on a FX96 Thermocycler (Bio- Rad). The PCR mixture contained TaqMan mix (Roche), $200 \mathrm{nM}$ of primers, the Universal Probe Library probe $(100 \mu \mathrm{M})$ for the gene of interest (TaqMan Gene Expression Assays [Primers/ probe]; Life technologies), and $1.67 \mu \mathrm{l} \mathrm{cDNA}$ template. Reactions were performed at least in technical duplicates. The relative amount of mRNA was calculated using the comparative $\mathrm{Ct}(\triangle \Delta \mathrm{CT})$ method, following data normalization against GAPDH as a housekeeping gene. The PCR primers and UPL probes used for the qPCR are listed in Supplementary Fig. 1.

\section{Western blot}

Cells were directly lysed in Laemmli buffer. Cell lysates were resolved by SDS-PAGE electrophoresis and transferred to nitrocellulose membranes (Bio-Rad). Membranes were then blocked with TBST-Milk $5 \%$ for $1 \mathrm{~h}$ and incubated with primary antibodies overnight at $4{ }^{\circ} \mathrm{C}$. Primary antibodies used are listed in Supplementary Fig. 2. Horseradish peroxidase-conjugated donkey anti-rabbit and sheep anti-mouse antibodies (Interchim) were used as secondary antibodies and incubated for $1 \mathrm{~h}$ at room temperature. Detection was performed using the ECL kit (Amersham) and the Biorad Chemidoc system. 


\section{Comet assay}

For each condition, cells were suspended $1: 10$ in $0.5 \%$ low-melting point agarose at $37^{\circ} \mathrm{C}$. The suspension was immediately laid onto a Comet slide (Trevigen Inc.), 2.000 cells per well. Agarose was allowed to solidify at $4{ }^{\circ} \mathrm{C}$ for $15 \mathrm{~min}$. The Comet slides were then immersed in prechilled lysis solution (1.2 M NaCl, $100 \mathrm{mM}$ EDTA, $10 \mathrm{mM}$ Tris, 1\% Triton $(\mathrm{pH} \mathrm{10.0)})$ at $4{ }^{\circ} \mathrm{C}$ for $120 \mathrm{~min}$ in the dark. After this treatment, comet slides were allowed to equilibrate in electrophoresis buffer for $2 \times 15 \mathrm{~min}$ at $4{ }^{\circ} \mathrm{C}$ in the dark. The migration was performed in EDTA $2 \mathrm{mM} \mathrm{NaOH} 30 \mathrm{mM}$ ( $\mathrm{pH} 12.3$ ) buffer at $40 \mathrm{~V}$ for $15 \mathrm{~min}$. After migration, the slides were rinsed with water neutralized with $0.4 \mathrm{M}$ Tris ( $\mathrm{pH} 7.5$ ) and fixed $5 \mathrm{~min}$ in $70 \%$ ethanol. After the slides were stained with SYBR Safe (X1000; Invitrogen). Images were acquired with a Nikon fluorescence microscope. Tail moments ( $1 / 4$ tail length DNA in the tail/total DNA) were analyzed using the Casplab freeware ${ }^{27}$.

\section{Immunofluorescence staining}

Six days after infection in eight-chamber tissue culture slides (Falcon, Corning), cells were fixed in ice-cold methanol for $10 \mathrm{~min}$ at $-20^{\circ} \mathrm{C}$ and blocked in $0.05 \%$ PBSTween containing 20\% FBS (PBST-FBS). Incubation with primary antibodies in PBST-FBS was performed overnight at $4{ }^{\circ} \mathrm{C}$. Primary antibodies and dilutions used are listed in Supplementary Fig. 2. After three washes with PBS-Tween, the slides were incubated with Alexa Fluor 488 dye-conjugated goat anti-rabbit antibody or chicken anti-mouse antibody diluted in PBST-FBS for $1 \mathrm{~h}$ at room temperature. The slides were then washed with PBS-Tween and mounted with DAPI Fluoromount G (SouthernBiotech). Images were acquired with a Nikon fluorescence microscope. Number of foci per nucleus was determined using the Focinator tool ${ }^{28}$.

\section{Statistical analysis}

All statistical analysis and graphs, presented as mean between three independent experiments with SEM, were created with GraphPad Prism 7.03, unless indicated otherwise in figure legends. Two-tailed unpaired Student's $t$-test was used to determine the $p$ value unless indicated otherwise in the figure legends. ${ }^{*} p<0.05$; ${ }^{* * *} p<0.01$; ${ }^{* * * *} p<0.001$.

\section{Gene set enrichment analysis}

PLA2R1 co-expression correlation values and statistics for all genes of the genome were generated with the SEEK analysis tool (http://seek.princeton.edu/) using Multiple Tissue Profiling. Co-expression values were multiplied by the $p$ value to obtain ranked values. This preranked file was run with the GSEA Preranked module against CGP (Chemical and Genetic Perturbation) from Curated gene sets, against BP (Biological Pathway) from GO Gene sets or against Hallmark gene sets using default parameters.

\section{Mice}

C57/B6 PLA2R1 wild type and PLA2R1 knockout mice, both male and female, were used for experiments. Pla2r1 knockout mice were described and genotyped as in ref. ${ }^{29}$. Twelve and 20-month-old mice were sacrificed and tissues of interest removed and analyzed (macroscopic lesions, skin and spleen). Mice were maintained in a specific pathogen free (SPF) animal facility platform, Anican, at the Cancer Research Center of Lyon. The experiments were conducted in accordance with the animal care guidelines of the European Union and French laws. Protocols were approved by the local Animal Ethic Evaluation Committee (CECCAPP: C2EA-15) and authorized by the French Ministry of Education and Research.

\section{Immunohistochemistry}

Paraffin-embedded murine tissues were used. Slides were serially sectioned at $3 \mu \mathrm{m}$ thickness. After deparaffinization and rehydration, the slides were incubated in $3 \%$ hydrogen peroxide in distilled water to block endogenous peroxidases. For heat-induced antigen retrieval, tissue sections were boiled in $10 \mathrm{mmol} / \mathrm{L}$ citrate buffer $\mathrm{pH}$ 6.0 in a microwave oven for $15 \mathrm{~min}$. The slides were then incubated for $30 \mathrm{~min}$ with low-background antibody diluent (DAKO Real) to block unspecific antigen sites. They were then incubated at $4{ }^{\circ} \mathrm{C}$ overnight with the primary antibody (listed in Supplementary Fig. 2) diluted in the "low-background" antibody diluent (DAKO Real). After rinsing in PBS, the slides were incubated with a biotinylated secondary antibody bound to a streptavidin peroxidase conjugate (Dako E0468) for $1 \mathrm{~h}$ at room temperature. Slides were treated with Streptavidine HRP (Vector) and the bound antibody was then detected using the DAB peroxidase substrate kit (Vector). Sections were counterstained with hematoxylin and the slides were finally dehydrated and mounted. At least 500 cells taken from five independent fields were quantified per mouse. Mice were identified with a number and results were acquired without knowing the genotypes by the experimentator.

\section{Results}

Loss of Pla2r1 increases spontaneous tumor formation in aged mice

To investigate whether PLA2R1 regulates spontaneous tumor formation during aging, we generated mice cohorts composed of wild type (WT) and Pla2r1 knockout littermates. We sacrificed 12-month-old mice and examined skin, pancreas, colon, kidney, lung and mammary gland tissue sections. Two Pla2r1 KO mice displayed tumors, a lung adenoma, and a colon adenoma (Supplementary Fig. 3), suggesting an increased sensibility of Pla2r1 KO mice to develop tumors with aging. To confirm and extend 


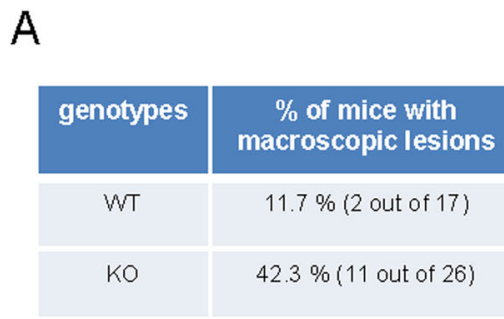

C
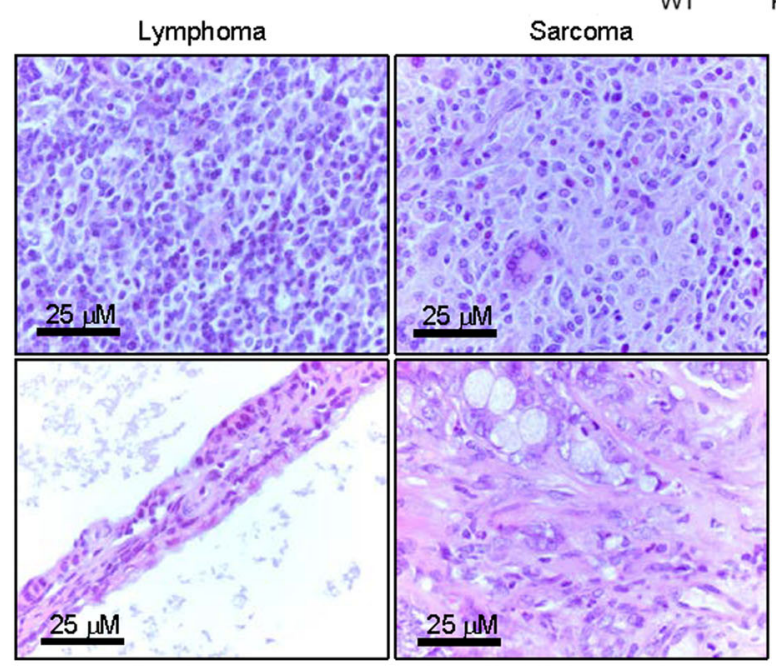

Intestinal Adenocarcinoma

D

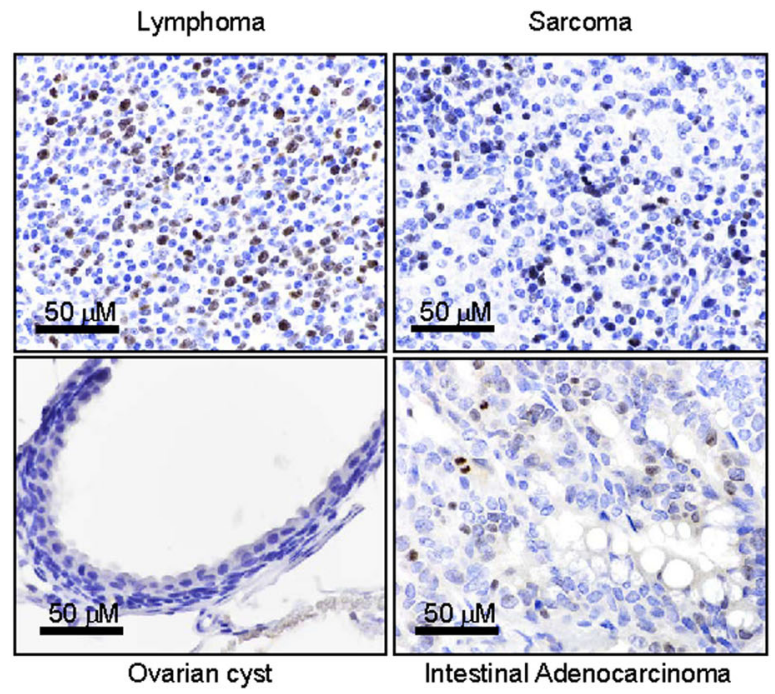

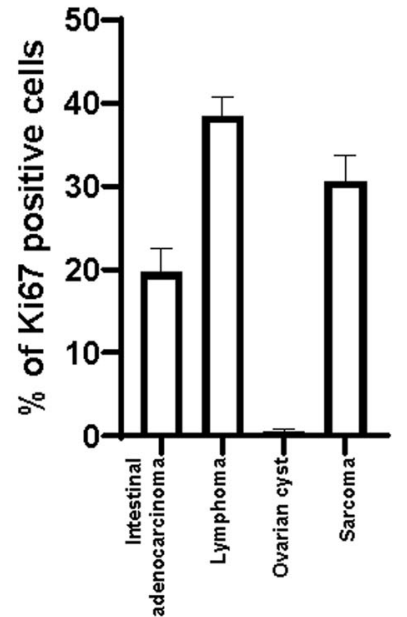

Fig. 1 Pla2r1 loss increases spontaneous tumor formation in old mice. A-C Macroscopic lesions of 20-month-old Pla2r1-/- mice ( $n=26)$ and their control littermates $(n=17)$ were counted and analyzed histologically to characterize each lesion. A Percentage (\%) of mice displaying macroscopic lesions for each genotype was calculated. $P=0.04$ according to two-tailed Fisher exact test. $\mathbf{B}$ The mean number of lesions per mouse for each genotype was computed and presented as mean \pm SEM. The $p$ value was calculated using Mann-Whitney test. ${ }^{*} p<0.05$. C Representative histopathological images of lesions are shown. $\mathbf{D}$ Indicated tumors were prepared and analyzed by immunohistochemistry against Ki67 proliferation marker to determine the percentage of positive cells. Representative images and histograms are shown (mean \pm SD). 
these results older mice were sacrificed at 20 months, prior to conducting macroscopic analyses. Approximately half of the Pla2r1 KO mice displayed macroscopic lesions, whereas only $11 \%$ of the control littermates displayed such lesions (Fig. 1A). Moreover, the number of lesions per mouse was also higher in $\mathrm{KO}$ mice (Fig. 1B). Anatomopathological examination of these lesions confirmed their tumoral origin, though both benign and malignant lesions were observed (Table 1, Fig. 1C and Supplementary Fig. 4), malignant lesions displaying higher Ki67 proliferation marker staining when compared to benign lesions (Fig. 1D). Anatomo-pathological examination on tissue sections of breast, kidney, pancreas, lung, colon, liver and thymus did not reveal additional increase of other neoplastic lesions in 20-month-old KO mice when compared to WT littermates. These results suggest that Pla2r1 protects mice from tumor formation during aging.

PLA2R1 expression is correlated with loss of a DNA repair signature, decreased PARP1 expression, and increased DNA damage

Accumulation of DNA damage is known to be induced by oncogenic signals where it can contribute to tumor suppression through activation of its downstream signaling resulting in p53 activation ${ }^{4,30}$. PLA2R1 has been shown to regulate DNA damage accumulation in vitro ${ }^{16}$. Still little is known about the molecular, cellular, and physiological functions of PLA2R1 in humans ${ }^{14}$. To gain further insight into its function, we explored the computational gene co-expression search engine SEEK (Search-Based Exploration of Expression Compendium) which contains massive human expression compendium that contains thousands of expression datasets to define genes and pathways co- or inversely expressed with PLA2R1. By this means, we obtained a full list of genes correlated or inversely correlated with PLA2R1 expression (Supplementary Table 1), and subsequently used this list to perform Gene Set Enrichment Analysis (GSEA). These analyses revealed a strong inverse correlation between PLA2R1 levels and the expression of a DNA repair signature (Fig. 2A). PARP1 was one of the top-ranking inversely correlated genes uncovered in that signature, as well as globally in the whole transcriptome (Fig. 2B and Supplementary Table 1). PARP1 is involved in DNA repair $^{31}$. It is known to be downregulated during senescence and when ectopically expressed is able to overcome DNA damage and senescence, furthermore its inhibition promotes cell death ${ }^{12,32}$. PLA2R1 exerts quite opposite effects as it is induced during senescence ${ }^{16}$ and its downregulation decreases DNA damage and senescence $^{16}$, as well as it promotes cell death when ectopically expressed $^{17}$. Because of inverse correlation between these two genes and opposition in their functional effects, we further explored the link between PLA2R1 and PARP1 by
Table 1 Macroscopic lesions observed in 20-monthold mice.

\begin{tabular}{|c|c|}
\hline $\begin{array}{l}\text { Genotype } \\
\text { and gender }\end{array}$ & Lesion type \\
\hline WT-F & Benign serous ovarian cyst \\
\hline WT-M & Lymphoma \\
\hline $\mathrm{KO}-\mathrm{F}$ & Ovarian cystadenoma \\
\hline $\mathrm{KO}-\mathrm{F}$ & Benign serous ovarian cyst \\
\hline \multirow[t]{3}{*}{$\mathrm{KO}-\mathrm{M}$} & Ovarian cystadenoma \\
\hline & Lymphoma \\
\hline & Metastatic lymphoma in the liver \\
\hline $\mathrm{KO}-\mathrm{F}$ & $\begin{array}{l}\text { Periportal multifocal invasion by lymphoid } \\
\text { proliferation in the liver. Probably lymphoma }\end{array}$ \\
\hline $\mathrm{KO}-\mathrm{F}$ & $\begin{array}{l}\text { Periportal multifocal invasion by lymphoid } \\
\text { proliferation in the liver. Probably lymphoma }\end{array}$ \\
\hline $\mathrm{KO}-\mathrm{M}$ & Lymphoma \\
\hline $\mathrm{KO}-\mathrm{M}$ & Intestinal adenocarcinoma \\
\hline \multirow[t]{5}{*}{$\mathrm{KO}-\mathrm{M}$} & Lymphoma and hystiocytic sarcoma \\
\hline & Lymphoma \\
\hline & Lymphoma \\
\hline & Lymphoma \\
\hline & Lymphoma \\
\hline $\mathrm{KO}-\mathrm{F}$ & Benign serous ovarian cyst \\
\hline $\mathrm{KO}-\mathrm{F}$ & Benign serous ovarian cyst \\
\hline $\mathrm{KO}-\mathrm{F}$ & Benign serous ovarian cyst \\
\hline
\end{tabular}

examining the level of Parp1 in WT and Pla2r1 KO mice. We found that 20-month-old Pla2r1 KO mice had a tendency toward higher levels of Parp1 (Fig. 2C) and displayed less DNA damage according to the decreased percentage of $\gamma \mathrm{H} 2 \mathrm{AX}$-positive cells (Fig. 2D). These differences were not seen in 12-month-old mice (Supplementary Fig. 5), suggesting that these differences took place with aging. In addition, an increase in Parp1 was correlated with a decrease in $\gamma \mathrm{H} 2 \mathrm{AX}$ levels in old mice (Fig. 2E). Next, PLA2R1 was constitutively expressed in normal human cells, where it is known to induce premature senescence and DNA damage ${ }^{16}$. Its constitutive expression decreased both PARP1 mRNA and protein levels (Fig. 2F-H).

Overall, these results indicate that PLA2R1 is involved in the reduction of expression of the DNA repair factor, PARP1.

\section{PARP1 constitutive expression rescues PLA2R1-induced DNA damage and senescence}

Having shown that expression of PARP1 is reduced when PLA2R1 is increased, we wondered what the 
A

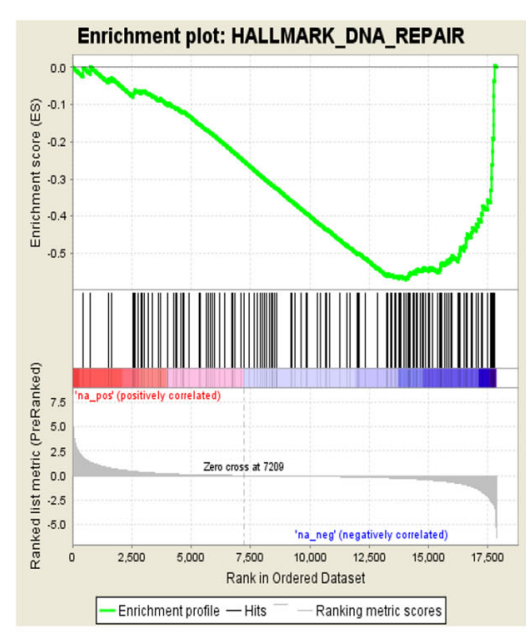

B

Coexpression score with PLA2R1

\begin{tabular}{|c|c|}
\hline Description & $\begin{array}{c}\text { poly (ADP-ribose) } \\
\text { polymerase 1 }\end{array}$ \\
\hline Rank & 17847 (over 17857) \\
\hline Gene & PARP1 \\
\hline Coexpression Score & -1.3883 \\
\hline P-Value & 0.0026 \\
\hline
\end{tabular}

E

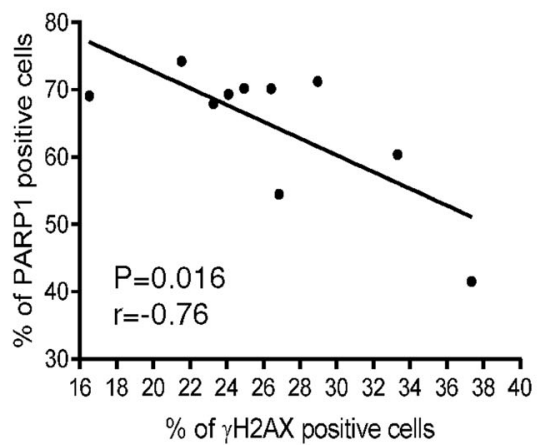

G

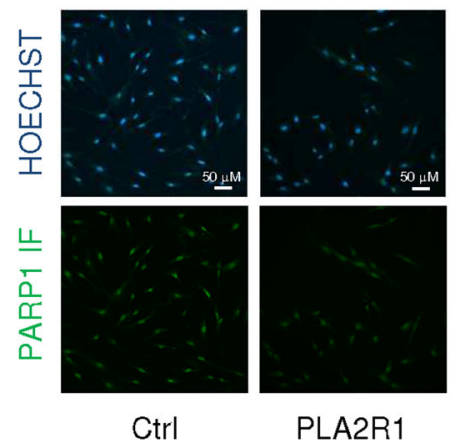

C

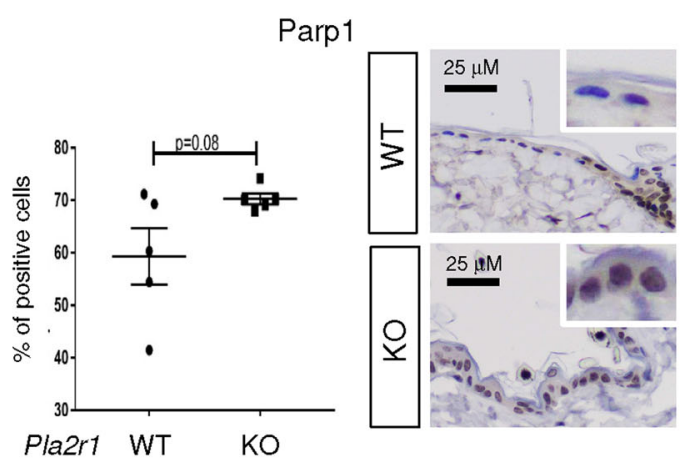

D
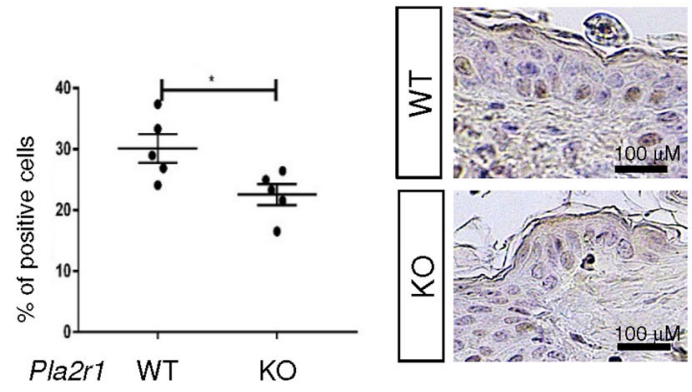

F

PLA2R1
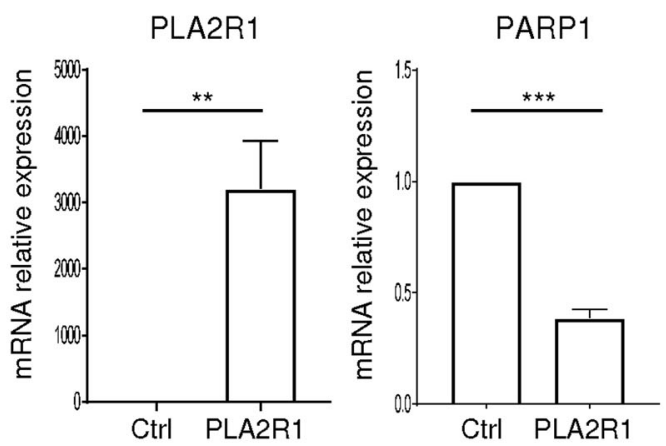

$\mathrm{H}$

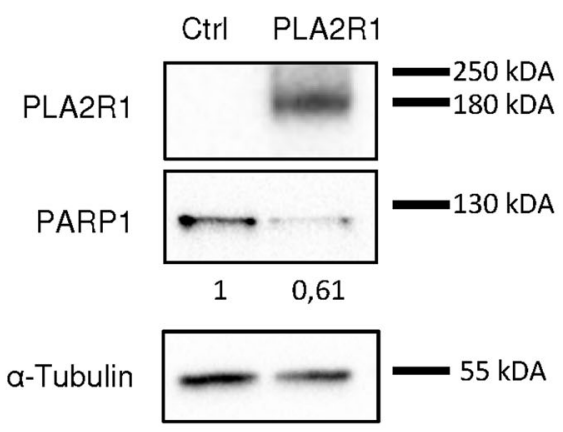

Fig. 2 (See legend on next page.) 
(see figure on previous page)

Fig. 2 PLA2R1 represses DNA repair gene PARP1 and correlates with DNA damage. A Correlation between the expression of all genes of the genome and PLA2R1 in human samples was generated using SEEK analysis tool (http://seek.princeton.edu/). Gene Set Enrichment Analysis (GSEA) was used to investigate signatures showing a correlation with PLA2R1 expression. Graph displays negative enrichment of the gene set "DNA repair hallmark" in PLA2R1 correlated genes (FDR q val < 0.001). B Table displays the inverse correlation between the expressions of PARP1 and PLA2R1 using the SEEK analysis tool. C, D Skin from 20-month-old WT $(n=5)$ and Pla2r1 KO $(n=5)$ mice was prepared and analyzed by

immunohistochemistry against $\mathrm{YH} 2 \mathrm{AX}(\mathbf{C})$ or PARP1 to determine the percentage of Parp1-positive cells (D). Results are presented as mean \pm SEM. $P$ value was calculated using Student's $t$-test. ${ }^{*} p<0.05$. E Correlation between proportion of PARP1-positive cells and $\gamma H 2 A X$-positive cells in the skin of 20-month-old mice $(n=10)$. Pearson test was used for statistical analysis. F-H MRC5 normal human cells were infected with a control or a PLA2R1encoding retroviral vector and selected. Between 5 and 7 days after infection, $\mathbf{F}$ RNA was prepared and RTqPCR performed against PLA2R1 or PARP1 and normalized against GAPDH levels. Results are shown as mean \pm SEM $(n=6)$. $P$ value was calculated using Student's $t$-test. ${ }^{* *} p<0.01$; ${ }^{* * *} p<0.001$. G Cells were fixed in methanol and immunofluorescence staining against PARP1 was performed. Nucleus counterstaining using Hoechst dye was performed. Representative images of three experiments are shown. $\mathbf{H}$ Cell extracts were prepared and western blot analysis was performed using the indicated antibodies. Quantification for PARP1 levels normalized against tubulin was performed with ImageJ. Representative images of three

experiments are shown.

functional consequences of this reduction were on PLA2R1-induced DNA damage and could we revert it. To address this issue, we performed rescue experiments by ectopically expressing PARP1 in PLA2R1 constitutively expressing normal human cells (Fig. 3A).

Quantitative analysis of DNA damage was performed by examining recruitment of 53BP1 and recruitment/phosphorylation of $\gamma \mathrm{H} 2 \mathrm{AX}$ factors at DNA damaged sites. As expected, increased PLA2R1 expression induced an augmentation in the number of both 53BP1 and $\gamma \mathrm{H} 2 \mathrm{AX}$ foci, which were reverted by the ectopic expression of PARP1 (Fig. 3B, C). Furthermore, Comet assay to directly measure DNA breaks confirmed that PLA2R1 constitutive expression induced DNA damage, whereas PARP1 rescued this PLA2R1 effect (Fig. 3D). As PARP1 rescued PLA2R1-induced DNA damage, we examined whether this also rescued PLA2R1-induced cellular senescence. Indeed, the ectopic expression of PARP1 rescued PLA2R1-induced growth arrest (Fig. 4A-C) and PLA2R1induced senescence markers, namely SA- $\beta$-Gal activity (Fig. 4D) as well as the increased expression of the p53 target, p21, and of IL8 (Fig. 4E, F).

Altogether, these results support that PARP1 decrease is critical for the mediation of the PLA2R1 effects on DNA damage and senescence.

\section{PARP1 transcription is repressed by a ROS-Rb pathway in PLA2R1-expressing cells}

Next, we investigated the mechanism leading to a decrease in PARP1 levels following PLA2R1 signaling. ROS are key mediators of DNA damage and cellular senescence $^{33}$. They have previously been described to be involved in the downregulation of PARP1 in the context of senescence ${ }^{12}$. Although how they are mediating PARP1 downregulation remains unknown. Moreover, ROS and the ensuing response are induced by PLA2R1 and are involved in PLA2R1-mediated senescence or cell death (Fig. 5A $)^{16,17}$. In addition, human dataset analysis showed that PLA2R1 expression was strongly correlated with the GO signature "Response to oxidative stress" (Supplementary Fig. 6) further supporting a link between PLA2R1 and ROS production. To examine whether ROS induced by PLA2R1 are involved in the repression of PARP1, we treated PLA2R1 ectopically expressing cells with two different antioxidants NAC and Trolox. Both compounds decreased PARP1 downregulation induced by PLA2R1 (Fig. 5B). Furthermore, $\mathrm{H}_{2} \mathrm{O}_{2}$ treatment mimicked the constitutive expression of PLA2R1 as it decreased PARP1 levels (Fig. 5C). Using human datasets, we found that PLA2R1 expression was inversely correlated with the signature "E2F targets" (Fig. 5D). Rb inhibits E2F transcriptional activities and participates in PLA2R1-induced senescence ${ }^{16,17}$. Rb and E2F transcription factors are both master regulators of cellular senescence and of the expression of DNA repair genes ${ }^{34}$. We identified that $\mathrm{Rb}$ was bound to the PARP1 promoter in senescent cells (Fig. $5 \mathrm{E})$, according to chromatin immunoprecipitation seq experiments $^{35}$. Knockdown of $\mathrm{Rb}$ reverted PARP1 decrease induced by $\mathrm{H}_{2} \mathrm{O}_{2}$ treatment (Fig. $5 \mathrm{C}$ ) or by the constitutive expression of PLA2R1 (Fig. 5F), further highlighting a role for $\mathrm{Rb}$ in repressing PARP1 expression in senescent cells.

Hence, our results support the hypothesis that $\mathrm{Rb}$ directly represses PARP1 expression during PLA2R1ROS-induced senescence.

\section{Discussion}

In this study, we demonstrated the role of PLA2R1 in tumor suppression during aging. First and foremost, loss of Pla2r1 in mice resulted in increased tumor formation in old animals. This effect was most probably linked to decreased ROS, DNA damage, and DNA damage signaling. Mechanistically, PLA2R1 promotes repression of the PARP1 DNA repair protein, an increase in DNA damage and cellular senescence, which are dependent of a ROS-Rb pathway. 

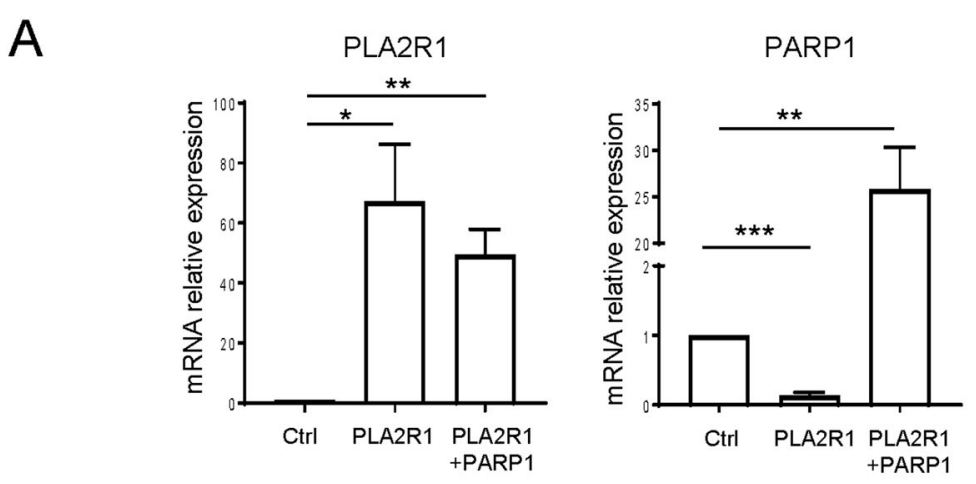

B
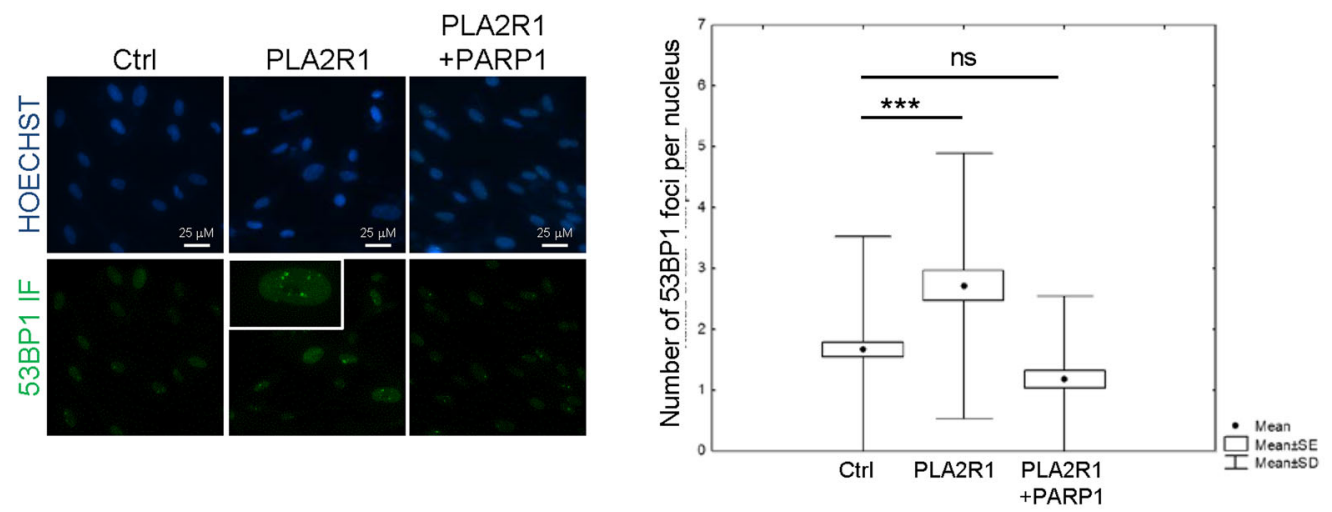

C
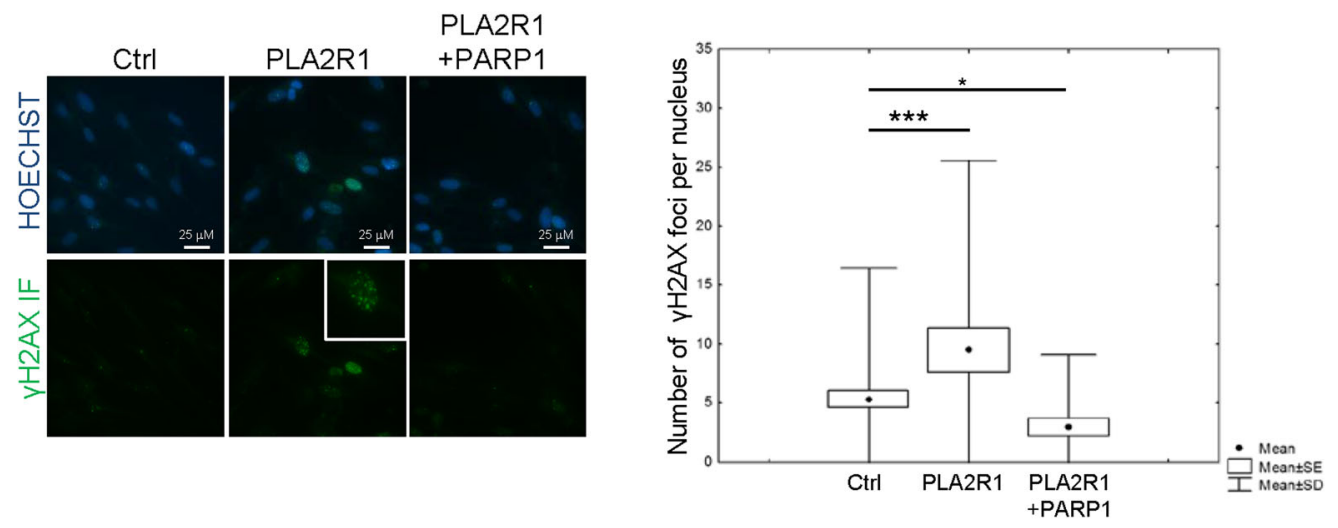

D

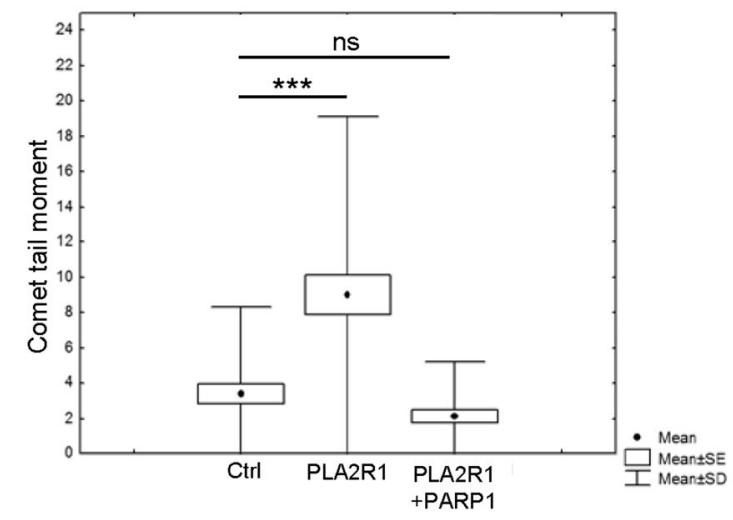

Fig. 3 (See legend on next page.) 
(see figure on previous page)

Fig. 3 PARP1 expression abolishes DNA damage induced by PLA2R1. A-D MRC5 normal human cells were infected with a control, PLA2R1-, or PARP1-encoding retroviral vectors as indicated and selected. A RNA was prepared and RTgPCR performed against PLA2R1 or PARP1 and normalized against GAPDH levels. The results shown are mean \pm SEM of three independent experiments. $P$ value was calculated using Student's $t$-test. ${ }^{*} p<0.05$; ${ }^{* *} p<0.01 ;{ }^{* * *} p<0.001$. B, C Cells were fixed in methanol and immunofluorescence staining against 53BP1 (B) and $\gamma \mathrm{H} 2 \mathrm{AX}(\mathbf{C})$ was performed with indicated antibodies. Images were taken using a Nikon Fluorescence microscope and quantified with the Focinator software. More than 50 nuclei were analyzed per condition. The $p$ value was calculated using the Mann-Whitney test. ${ }^{*} p<0.05 ;{ }^{* *} p<0.01 ;{ }^{* *} p<0.001$. D Comet assay for assessing DNA breaks was performed. After migration, the slides were stained with SYBR Safe. Tail moments (1/4 tail length DNA in the tail/total DNA) were analyzed using the Casplab software. A least 50 nuclei were analyzed per condition. The $p$ value was calculated using the Mann-Whitney test, ${ }^{* * *} p<0.001$.
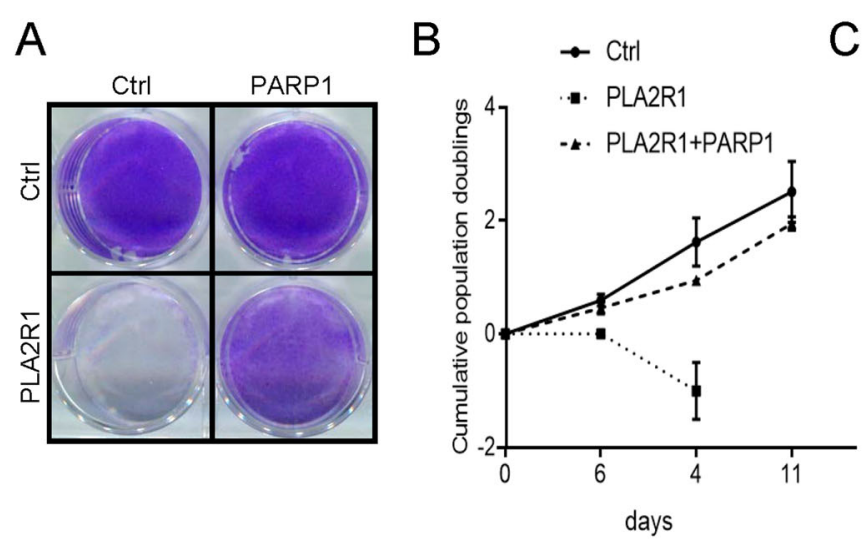

D

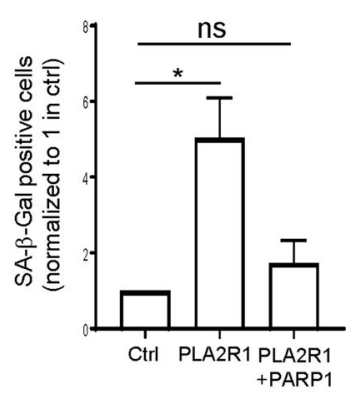

E

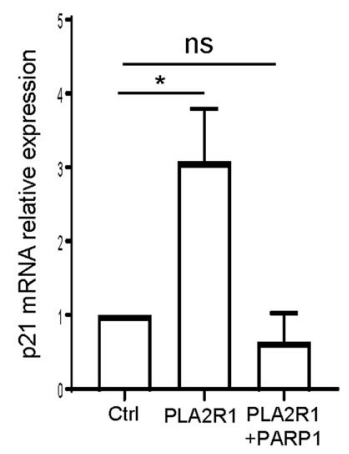

C

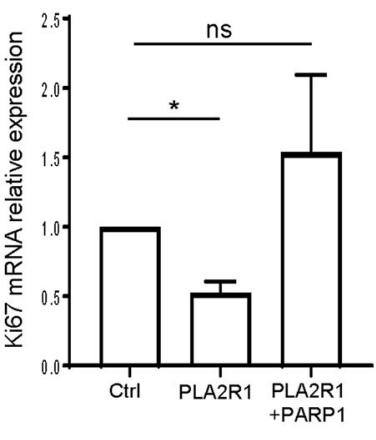

PLA2R1 +PARP1

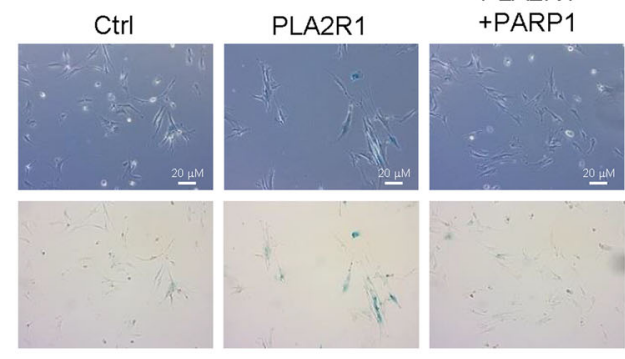

F

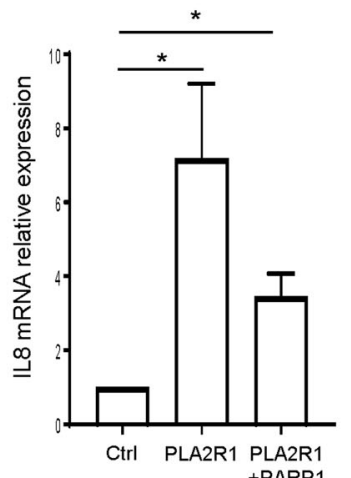

Fig. 4 PARP1 expression inhibits senescence induced by PLA2R1. E-F MRC5 cells were infected with control, PLA2R1-, or PARP1-encoding retroviral vectors as indicated, and selected. A Fourteen days after seeding, cells were fixed and stained by crystal violet to visualize cell density. B The growth curve was performed by counting the number of cells and calculating the population doublings at each cell passage. Representative growth curves from three experiments with SD are shown. C RNA was prepared and RTqPCR performed against the proliferation marker Ki67 and normalized against GAPDH levels $(n=3)$. D Cells were fixed and a SA- $\beta$-galactosidase assay was performed. Percentage of SA- $\beta$-Gal positive cells was calculated and representative images are shown $(n=3)$. E, F RTqPCR performed against p21 (E) and IL8 (F) and normalized against GAPDH levels $(n=3)$. Graphs $\mathbf{C}-\mathbf{F}$ show mean between three experiments \pm SEM and $p$ value was calculated using Student's $t$-test. ${ }^{*} p<0.05$. 

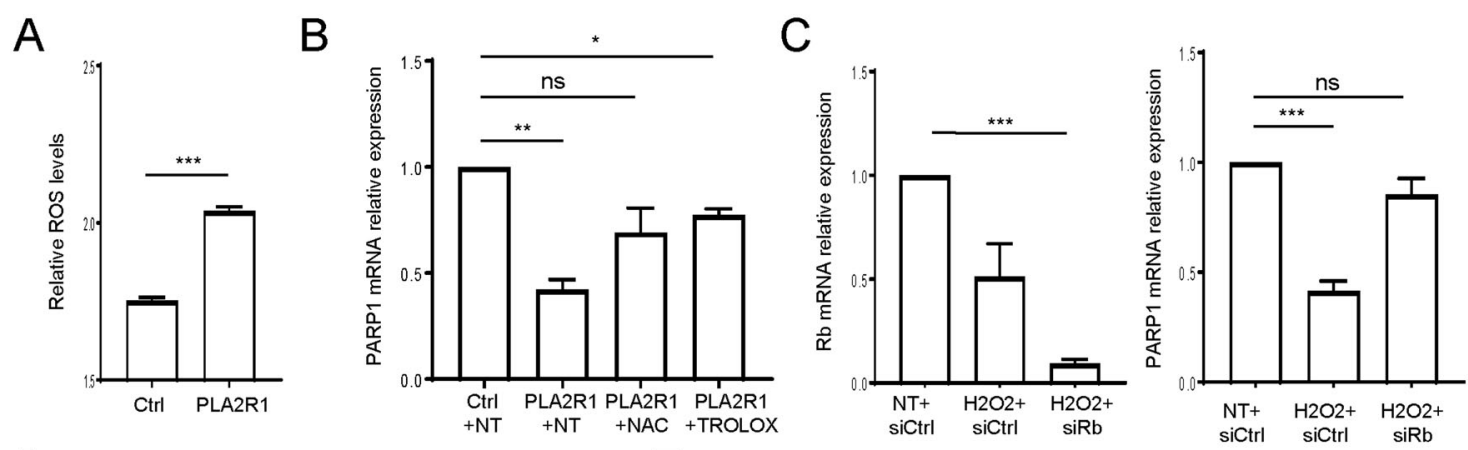

D

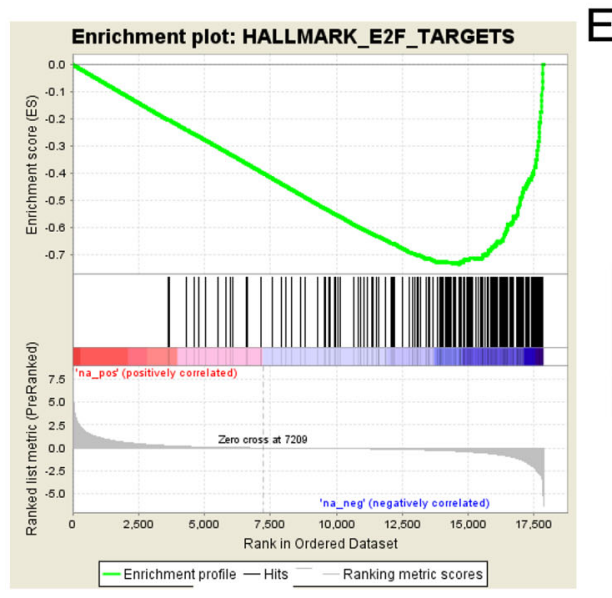

E

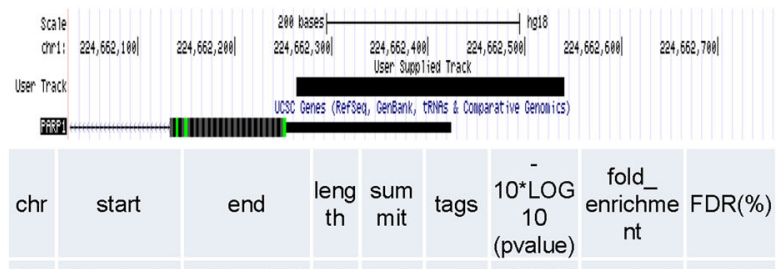

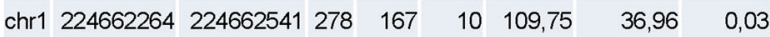

$\mathrm{F}$
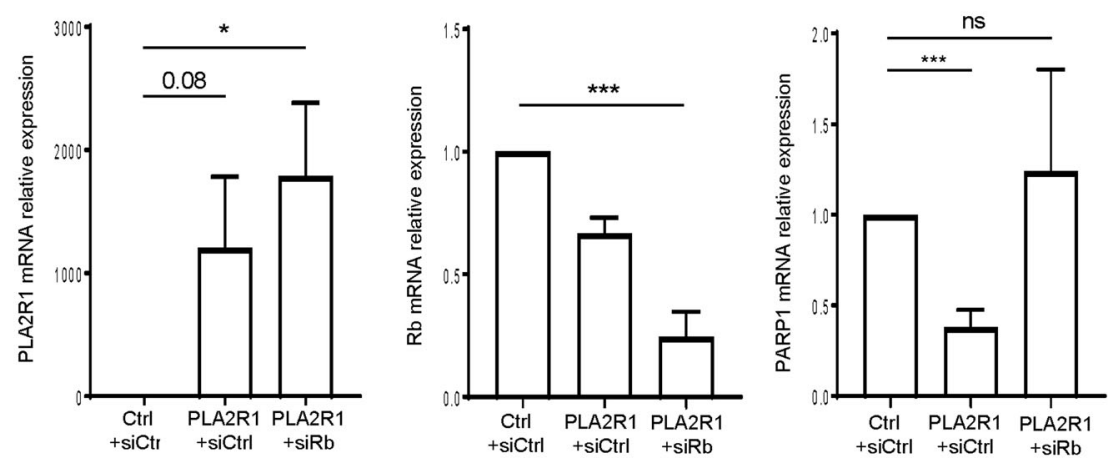

Fig. 5 PARP1 expression is repressed by a ROS/Rb pathway. A MRC5 cells were infected with roGFP2-ORP1, a ratiometric ROS probe, encoding retroviral vector and a control or PLA2R1-encoding retroviral vectors and selected. Images were taken by Operetta system and quantified by Columbus software. The results are shown as representative from three experiments, mean \pm SEM between three wells. $P$ value was calculated using Student's $t$-test ${ }^{* * *} p<0.001$. B MRC5 cells were infected with a control or a PLA2R1-encoding retroviral vector and selected. Cells were treated with NAC or Trolox daily during 3 days before analysis. Levels of PARP1 were quantified by RTqPCR and normalized against the level of GAPDH. The results are shown as mean \pm SEM $(n=2)$. $P$ value was calculated using Student's $t$-test. ${ }^{*} p<0.05 ;{ }^{*} p<0.01$. C MRC5 cells where transfected with a control nontargeting pool (siCtrl) or siRNA pools targeting Rb. Two days after transfection cells were treated with $\mathrm{H}_{2} \mathrm{O}_{2}$; mRNA levels of Rb and PARP1 were quantified by RTaPCR $24 \mathrm{~h}$ later. Results were normalized against the level of GAPDH mRNA. The results shown are mean $\pm \operatorname{SEM}(n=4)$ and $p$ value was calculated using Student's $t$-test ${ }^{* *} p<0.01 ;{ }^{* * *} p<0.001$. D GSEA analysis was performed on PLA2R1 co-expressed genes. Graph displays negative enrichment of E2F target genes between PLA2R1 correlated genes (FDR $q$ val $<0.001$ ). E ChiP seq data ${ }^{35}$ against Rb on senescent human fibroblasts were analyzed to reveal a binding of Rb to the PARP1 promoter. $\mathbf{F}$ MRC5 cells where transfected with a control nontargeting pool (siCtrl) or siRNA pools targeting Rb. The following day, cells were infected with a control or a PLA2R1-encoding retroviral vector and selected. mRNA levels of PLA2R1 (left), Rb (middle), and PARP1 (right) were quantified by RTgPCR. Results were normalized against the level of GAPDH mRNA. Mean \pm SEM $(n=4)$ are shown and $p$ value was calculated using Student's t-test. ${ }^{*} p<0.05$; ${ }^{* * *} p<0.001$.

Even if we have focused on the relationship between PLA2R1 and PARP1, we can speculate that other DNA repair pathways are linked to PLA2R1 and its effects. Indeed, analysis of multiple human datasets using SEEK database support a strong anti-correlation between PLA2R1 levels and the Fanconi anemia pathway (Supplementary Table 2). 
The role of DNA damage on cancer initiation is complex. According to our results, PLA2R1 could protect cells from tumor initiation during aging by promoting DNA damage and its downstream signaling such as activation of p53. In the context of PLA2R1 and beyond, p53 activation could result in cell death or/and cellular senescence ${ }^{16,36}$. This corroborates previous observations that DNA damage response acts as an anti-tumoral barrier ${ }^{4,6}$. Nevertheless this initial anti-tumoral barrier could constitute a selective process, promoted by DNA instability associated with DNA damage, to produce gene mutations promoting escape from cell death and senescence and tumor formation, such as mutations affecting the p53 pathway ${ }^{7}$. Consistently, mutations or loss-of-functions of multiple DNA repair genes, which promote chronic DNA damage, cell death, senescence, and instability promote long-term tumor formation ${ }^{10-12,34,37}$. In the balance between pro-tumoral effects of DNA damage and subsequent genetic instability, and DNA damage signaling anti-tumoral effects, the latter seems to prevail in the context of PLA2R1.

We identified ROS as critical regulators of PLA2R1induced DNA damage. ROS are known to induce genomic instability and are often thought to exert adverse effects on tumor initiation and progression as well as on other age-related diseases ${ }^{8}$. These speculations support the rationale to add antioxidants in anti-aging pills, which are quite popular in today's society. Nevertheless, these ideas have been challenged as anti-oxidant treatments might promote tumor aggressiveness ${ }^{24,38,39}$. As accumulation of ROS in the context of PLA2R1 induces DNA damage, cell death and cellular senescence ${ }^{16,17,33}$ and as DNA damage and its signaling, cell death and senescence can display tumor suppressive effects, it raises the question of whether ROS could exert tumor suppressive effects, as suggested by our data. In that case, ROS could be viewed as a messenger driving DNA damage and its downstream pathway, such as p53, and subsequent cell death and cellular senescence to suppress tumor formation. In line with this speculation, some epidemiological studies suggest deleterious effect of anti-oxidant supplements in the context of some age-related diseases, including cancer ${ }^{40,41}$ and one functional study suggests that NAC anti-oxidant treatment in mice promotes tumor formation during normal and accelerated aging, while it reduces some other marks of aging ${ }^{5}$.

ROS-induced DNA damage is largely thought to be formed due to the direct attack of DNA by $\operatorname{ROS}^{42,43}$. Our results suggest a more complex view where defects in DNA repair capacity by decreasing PARP1 levels largely contribute to DNA damage levels. The active role of the loss of additional DNA repair genes has already been shown in different senescence contexts ${ }^{12,34}$. We can speculate that decreased expression of DNA repair genes, like PARP1, reinforces and sustains DNA damage and its tumor suppressive signaling which in turn prevents damaged cells to resume their proliferation and/or induce cell death. In addition, it is possible that a decreased PARP1 avoids error prone activity of re-ligation driven by PARP1 (refs. ${ }^{44,45}$ ), avoiding DNA instability and subsequent pro-tumoral effects.

Our findings also support that PLA2R1-ROS pathway activates $\mathrm{Rb}$, which can bind and repress PARP1 gene expression. The underlying mechanism regulating $\mathrm{Rb}$ activation by ROS may be mediated by the induction of cyclin-dependent kinase inhibitor such as p16 and p21 or other pathways ${ }^{46,47}$.

In conclusion, our work sheds light on the tumor suppressive effect of PLA2R1 during aging and its downstream effectors: ROS, PARP1, and DNA damage and its downstream tumor suppressive pathway. It also contributes to emphasizing the complex role of ROS and DNA damage that can exert both pro- and anti-tumoral effects.

\section{Acknowledgements}

We thank the laboratory members for helpful discussions.

\section{Funding}

This work was carried out with the support of the Association for International Cancer Research (AICR-11-0722), the ANR, the INCA, the Ligue régionale contre le cancer, comité de la Savoie et de l'Allier. A.H. is supported by la Fondation pour la recherche médicale FRM.

\section{Author details}

${ }^{1}$ Centre de Recherche en Cancérologie de Lyon, Inserm U1052, CNRS UMR 5286, Centre Léon Bérard, Université de Lyon, Lyon, France. ${ }^{2}$ INSERM U1053 Bordeaux Research in Translational Oncology, University of Bordeaux, Bordeaux Cedex, France. ${ }^{3}$ Centre International de Recherche en Infectiologie, Inserm U1111, CNRS, UMR5308, École Normale Supérieure de Lyon, Université de Lyon, Université Claude Bernard Lyon 1, Lyon, France. ${ }^{4}$ Institut de Pathologie, Centre de Biologie Pathologie, CHRU de Lille, Faculté de Médecine, Université de Lille, Lille Cedex, France. ${ }^{5}$ Plateau cellules tissus, CHU de Bordeaux, Pessac, France

\section{Author contributions}

A.H. designed and performed the experiments, analyzed results, and wrote the manuscript. A.G. and D.V. designed and performed experiments and analyzed results. D.B. supervised, designed experiments, analyzed results, and wrote the manuscript. S.J., J-M.F., J-J.M, D.G., L.A., S.D., H.H-V., R.D., L.P., J.M., P.B., S.A., and P. $D$. designed experiments and/or performed experiments and/or analyzed results. All authors reviewed/edited the manuscript and approved its final version.

\section{Conflict of interest}

The authors declare no competing interests.

\section{Ethics statement}

The mouse experiments were conducted in accordance with the animal care guidelines of the European Union and French laws. Protocols were approved by the local Animal Ethic Evaluation Committee (CECCAPP: C2EA-15) and authorized by the French Ministry of Education and Research. 


\section{Publisher's note}

Springer Nature remains neutral with regard to jurisdictional claims in published maps and institutional affiliations.

Supplementary information The online version contains supplementary material available at https://doi.org/10.1038/s41419-021-03468-3.

Received: 24 March 2020 Revised: 16 January 2021 Accepted: 21 January 2021

Published online: 16 February 2021

\section{References}

1. Smetana, K. Jr et al. Ageing as an important risk factor for cancer. Anticancer Res. 36, 5009-5017 (2016).

2. Schile, A., Dion, S., Imai-Leonard, D. \& Rosalinda Doty. Spontaneous tumors in aging colonies of C57BL/6J mice. Cancer Res. 78, (13 Suppl.) Abstract nr 5113 (2018).

3. Loft, S. \& Poulsen, H. E. Cancer risk and oxidative DNA damage in man. J. Mol. Med. (Berl.) 74, 297-312 (1996).

4. Bartkova, J. et al. DNA damage response as a candidate anti-cancer barrier in early human tumorigenesis. Nature 434, 864-870 (2005).

5. Breau, M. et al. The antioxidant N-acetylcysteine protects from lung emphysema but induces lung adenocarcinoma in mice. JCl Insight 4, e127647 (2019).

6. Di Micco, R. et al. Oncogene-induced senescence is a DNA damage response triggered by DNA hyper-replication. Nature 444, 638-642 (2006).

7. Gorgoulis, V. G. et al. Activation of the DNA damage checkpoint and genomic instability in human precancerous lesions. Nature 434, 907-913 (2005).

8. Willcox, J. K., Ash, S. L. \& Catignani, G. L. Antioxidants and prevention of chronic disease. Crit. Rev. Food Sci. Nutr. 44, 275-295 (2004).

9. Maynard, S., Schurman, S. H., Harboe, C., de Souza-Pinto, N. C. \& Bohr, V. A. Base excision repair of oxidative DNA damage and association with cancer and aging. Carcinogenesis 30, 2-10 (2009).

10. Bogliolo, M. et al. The Fanconi anaemia genome stability and tumour suppressor network. Mutagenesis 17, 529-538 (2002).

11. Jasin, M. Homologous repair of DNA damage and tumorigenesis: the BRCA connection. Oncogene 21, 8981-8993 (2002).

12. Nassour, J. et al. Defective DNA single-strand break repair is responsible for senescence and neoplastic escape of epithelial cells. Nat. Commun. 7, 10399 (2016).

13. Weaver, B. A., Silk, A. D., Montagna, C., Verdier-Pinard, P. \& Cleveland, D. W. Aneuploidy acts both oncogenically and as a tumor suppressor. Cancer Cell 11, 25-36 (2007).

14. Sukocheva, O. et al. Current insights into functions of phospholipase A2 receptor in normal and cancer cells: more questions than answers. Semin. Cancer Biol. 56, 116-127 (2019).

15. Bernard, D. \& Vindrieux, D. PLA2R1: expression and function in cancer. Biochim. Biophys. Acta 1846, 40-44 (2014).

16. Augert, A. et al. The M-type receptor PLA2R regulates senescence through the p53 pathway. EMBO Rep. 10, 271-277 (2009).

17. Augert, A. et al. PLA2R1 kills cancer cells by inducing mitochondrial stress. Free Radic. Biol. Med. 65C, 969-977 (2013).

18. Griveau, A. et al. The PLA2R1-JAK2 pathway upregulates ERRalpha and its mitochondrial program to exert tumor-suppressive action. Oncogene $\mathbf{3 5}$, 5033-5042 (2016)

19. Vindrieux, D. et al. PLA2R1 mediates tumor suppression by activating JAK2. Cancer Res. 73, 6334-6345 (2013).

20. Friedemann, M. et al. Methylation of the phospholipase A2 receptor 1 promoter region in childhood B cell acute lymphoblastic leukaemia. Sci. Rep. 10, 9058 (2020).

21. Vindrieux, D. et al. Repression of PLA2R1 by c-MYC and HIF-2alpha promotes cancer growth. Oncotarget 5, 1004-1013 (2014).
22. Griveau, A. et al. Targeting the phospholipase A2 receptor ameliorates premature aging phenotypes. Aging Cell 17, e12835 (2018).

23. Wang, X., Michael, D., de Murcia, G. \& Oren, M. p53 activation by nitric oxide involves down-regulation of Mdm2. J. Biol. Chem. 277, 15697-15702 (2002).

24. Le Gal, K. et al. Antioxidants can increase melanoma metastasis in mice. Sci. Transl. Med. 7, 308re8 (2015).

25. Dimri, G. P. et al. A biomarker that identifies senescent human cells in culture and in aging skin in vivo. Proc. Natl Acad. Sci. USA 92, 9363-9367 (1995).

26. Gutscher, M. et al. Proximity-based protein thiol oxidation by $\mathrm{H}_{2} \mathrm{O}_{2}$-scavenging peroxidases. J. Biol. Chem. 284, 31532-31540 (2009).

27. Konca, K. et al. A cross-platform public domain PC image-analysis program for the comet assay. Mutat. Res. 534, 15-20 (2003).

28. Oeck, S., Malewicz, N. M., Hurst, S., Rudner, J. \& Jendrossek, V. The Focinator-a new open-source tool for high-throughput foci evaluation of DNA damage. Radiat. Oncol. 10, 163 (2015).

29. Hanasaki, K., Yokota, Y., Ishizaki, J., Itoh, T. \& Arita, H. Resistance to endotoxic shock in phospholipase A2 receptor-deficient mice. J. Biol. Chem. 272, 32792-32797 (1997)

30. Bartkova, J. et al. Oncogene-induced senescence is part of the tumorigenesis barrier imposed by DNA damage checkpoints. Nature 444, 633-637 (2006).

31. Caldecott, K. W. Protein ADP-ribosylation and the cellular response to DNA strand breaks. DNA Repair (Amst.) 19, 108-113 (2014).

32. Beneke, S., Diefenbach, J. \& Burkle, A. Poly(ADP-ribosyl)ation inhibitors: promising drug candidates for a wide variety of pathophysiologic conditions. Int J. Cancer 111, 813-818 (2004).

33. Lu, T. \& Finkel, T. Free radicals and senescence. Exp. Cell Res. 314, 1918-1922 (2008).

34. Collin, G., Huna, A., Warnier, M., Flaman, J. M. \& Bernard, D. Transcriptional repression of DNA repair genes is a hallmark and a cause of cellular senescence. Cell Death. Dis. 9, 259 (2018).

35. Chicas, A. et al. Dissecting the unique role of the retinoblastoma tumor suppressor during cellular senescence. Cancer Cell 17, 376-387 (2010).

36. Papazoglu, C. \& Mills, A. A. p53: at the crossroad between cancer and ageing. J. Pathol. 211, 124-133 (2007).

37. Sedic, M. et al. Haploinsufficiency for BRCA1 leads to cell-type-specific genomic instability and premature senescence. Nat. Commun. 6, 7505 (2015).

38. Piskounova, E. et al. Oxidative stress inhibits distant metastasis by human melanoma cells. Nature 527, 186-191 (2015).

39. Sayin, V. I. et al. Antioxidants accelerate lung cancer progression in mice. Sci. Transl. Med. 6, 221 ra15 (2014).

40. Albanes, D. et al. Alpha-tocopherol and beta-carotene supplements and lung cancer incidence in the alpha-tocopherol, beta-carotene cancer prevention study: effects of base-line characteristics and study compliance. J. Nat/ Cancer Inst. 88, 1560-1570 (1996).

41. Bjelakovic, G., Nikolova, D., Gluud, L. L., Simonetti, R. G. \& Gluud, C. Mortality in randomized trials of antioxidant supplements for primary and secondary prevention: systematic review and meta-analysis. JAMA 297, 842-857 (2007).

42. Chen, J. H., Hales, C. N. \& Ozanne, S. E. DNA damage, cellular senescence and organismal ageing: causal or correlative? Nucleic Acids Res. 35, 7417-7428 (2007).

43. Rai, P. et al. Continuous elimination of oxidized nucleotides is necessary to prevent rapid onset of cellular senescence. Proc. Natl Acad. Sci. USA 106, 169-174 (2009).

44. Huang, $Y$. et al. Poly(ADP-ribose) polymerase-1 promotes recruitment of meiotic recombination-11 to chromatin and DNA double-strand break repair in Ku70-deficient breast cancer cells. FASEB J. fj201800092R (2018).

45. Muvarak, N. et al. c-MYC generates repair errors via increased transcription of alternative-NHEJ dactors, LIG3 and PARP1, in tyrosine kinase-activated leukemias. Mol. Cancer Res. 13, 699-712 (2015).

46. Takahashi, A. et al. Mitogenic signalling and the p16INK4a-Rb pathway cooperate to enforce irreversible cellular senescence. Nat. Cell Biol. 8, 1291-1297 (2006).

47. Vurusaner, B., Poli, G. \& Basaga, H. Tumor suppressor genes and ROS: complex networks of interactions. Free Radic. Biol. Med. 52, 7-18 (2012). 